\title{
Hydrophobicity of Biofilm Coatings Influences the Transport Dynamics of Polystyrene Nanoparticles in Biofilm-coated Sand
}

\author{
Submitted to: \\ Water Research
}

\begin{abstract}
MICHAEL R. MITZEL ${ }^{1,2}$, STEFANIE SAND ${ }^{1,3}$, JOANN K. WHALEN ${ }^{2}$ and NATHALIE TUFENKJI $1^{*}$
\end{abstract}

\author{
${ }^{1}$ Department of Chemical Engineering, \\ ${ }^{2}$ Department of Natural Resource Sciences, \\ McGill University, Montreal, Quebec H3A OC5, Canada \\ ${ }^{3}$ Department of Water Science, \\ Universität Duisburg-Essen, \\ Universitätsstr. 5, 45141 Essen, Germany
}

*Corresponding Author. Phone: (514) 398-2999; Fax: (514) 398-6678; E-mail: nathalie.tufenkji@mcgill.ca 


\begin{abstract}
Engineered nanoparticles (ENPs) are used in the manufacture of over 2,000 industrial and consumer products to enhance their material properties and functions or to enable new nanoparticle-dependent functions. The widespread use of ENPs will result in their release to the subsurface and aquatic environments, where they will interact with indigenous biota. Laboratory column experiments were designed to understand the influence of two different Pseudomonas aeruginosa biofilms on the mobility of polystyrene latex nanoparticles in granular porous media representative of groundwater aquifers or riverbank filtration settings. The transport behavior of $20 \mathrm{~nm}$ carboxylate-modified (CLPs) and sulfate (SLPs) polystyrene latex ENPs suspended in $\mathrm{NaCl}$ or $\mathrm{CaCl}_{2}$ (1 and $10 \mathrm{mM}$ ionic strength, $\mathrm{pH}$ 7) was studied in columns packed with quartz sand coated with biofilms formed by two $P$. aeruginosa strains that differed in cell surface hydrophobicity (P. aeruginosa $9027^{\mathrm{TM}}$, relatively hydrophilic and P. aeruginosa PAO1, relatively hydrophobic). Biofilm-coated quartz sand retained more of the electrostaticallystabilized latex ENPs than clean, uncoated sand, regardless of the serotype. As IS increased, clear differences in the shape of the ENP breakthrough curves were observed for each type of biofilm coating. ENP breakthrough in the P. aeruginosa PAO1 biofilm-coated sand was generally constant with time whereby breakthrough in the $P$. aeruginosa 9027 biofilm-coated sand showed dynamic behavior. This indicates a fundamental difference in the mechanisms of ENP deposition onto hydrophilic or hydrophobic biofilm coatings due to the hydration properties of these biofilms. The results of this study demonstrate the importance of considering the surface properties of aquifer grain coatings when evaluating ENP fate in natural subsurface environments.
\end{abstract}

\title{
Keywords:
}

Nanoparticle; filtration; biofilm; hydrophobicity; transport, fate 


\subsection{Introduction}

Novel nanotechnology is already impacting our everyday life and there continues to be rapid increase in the use of engineered nanoparticles (ENPs) in the industrial and commercial sectors. ENPs currently have vital roles in electronics, coatings and cosmetics industries (Musee, 2011; Sabitha et al., 2012). Keller et al. (2013) estimated that more than $70 \%$ of all ENPs will end up in landfills, where they are susceptible to release into natural subsurface environments. Suspended ENPs that remain through water treatment may also be released to surface waters where their filtration in riverbanks becomes relevant (Brar et al., 2010). The transport and fate of ENPs in granular aquatic environments representative of riverbanks and groundwater aquifers is dependent on ENP physicochemical properties (Petosa et al., 2010; Cornelis et al., 2014), but what is not known is how physicochemical properties of the granular collector surfaces (aquifer grains) present in natural subsurface environments could affect the interaction with and retention of ENPs.

Sessile bacteria, or 'biofilms', are found on nearly all environmental surfaces. Natural biofilms are a complex formation of bacteria and other microorganisms that typically accumulate at a solid-liquid interface and are surrounded and protected in a self-produced matrix of hydrated extracellular polymeric substances (Flemming \& Wingender, 2010). Natural biofilms can alter the roughness, charge, hydrophobicity, and chemistry of the aquifer grain surfaces upon which they exist (Donlan, 2002). Most studies that examined the influence of biofilms on ENP transport indicate that the presence of biofilms generally increases the retention of ENPs in a water saturated granular matrix and this behavior is generally observed with electrostatically-stabilized ENPs (e.g., Leon-Morales et al., 2007; Tong et al., 2010; Lerner et al., 2012; Tripathi et al., 2012; Jiang et al., 2013; Xiao \& Wiesner, 2013), but not necessarily with sterically-stabilized 
ENPs (Xiao \& Wiesner, 2013; Mitzel \& Tufenkji, 2014). For instance, Tong et al. (2010) found that Escherichia coli biofilm on quartz sand grains increased the deposition of nano-sized fullerene particles suspended in $\mathrm{NaCl}$ or $\mathrm{CaCl}_{2}$ solution. A similar result was reported by Tripathi et al. (2012), using sulfate polystyrene latex particles (SLPs) and carboxylate-modified polystyrene latex particles (CLPs) suspended in potassium chloride $(\mathrm{KCl})$ solution. However, some recent evidence suggests that biofilm coatings on grain surfaces may lead to enhanced transport of ENPs that are sterically-stabilized (Xiao \& Wiesner, 2013; Mitzel and Tufenkji, 2014). Xiao and Wiesner (2013) found significant retention of various ENPs by biofilms, but no difference in the retention of sterically-stabilized nano-silver $(n \mathrm{Ag})$ in the presence of biofilm, as compared to clean sand. Using a biofilm coating method that reduces the contribution of physical straining within the column, Mitzel and Tufenkji (2014) observed significantly greater mobility of sterically-coated $n \mathrm{Ag}$ in the presence of biofilms, as compared to clean sand. Thus, the related but limited literature suggests that biofilms can be considered a retentive factor for electrostatically-stabilized ENPs, but may lead to enhanced mobility of ENPs that are stericallystabilized.

Although previous research has investigated the role of different physicochemical properties of various electrostatically- or sterically-stabilized ENPs on their transport behavior in biofilm-coated sand, the role of hydrophobicity of the ENP surface and the biofilm remains unclear. Theory would indicate that hydrophobic particles should show greater attraction, and thereby greater attachment, to hydrophobic collector surfaces (Israelachvili \& Pashley, 1982; Chandler, 2005). Also, two hydrophilic surfaces would be expected to be repulsed by one another via hydration forces (Israelachvili \& Wennerström, 1996), although recent work provides evidence of attraction between hydrophilic surfaces (Kanduč et al., 2014). Xiao and 
Wiesner (2013) investigated the transport of different nAg and carbon-based ENPs through columns packed with glass beads coated with P. aeruginosa (ATCC \#7700) or B. cereus (ATCC \#14179), along with proteins and polysaccharides, to explore the influence of collector surface hydrophobicity. Xiao and Wiesner (2013) concluded that increasing particle and/or collector surface hydrophobicity leads to increased particle attachment efficiency. Song et al. (2011) also investigated the transport of $\mathrm{nAg}$ in columns packed with clean (hydrophilic) or octadecylichlorosilane (OTS)-coated (hydrophobic) glass beads. The nAg particles were prepared with three different coatings (citrate, polyvinylpyrrolidone, and gum arabic) that produced different degrees of surface hydrophobicity. Song et al. (2011) suggested that hydrophobic interactions between the gum arabic-coated nAg and the OTS-coated glass beads were responsible for the increased attachment of $\mathrm{nAg}$ with greater hydrophobicity to collectors with greater hydrophobicity. The results of previous studies may indicate a significant role for both ENP and collector surface hydrophobicity in the transport of ENPs within water saturated environments. However, with only a paucity of fundamental studies examining the impact of particle and collector surface hydrophobicity, more research in this area is needed. Experimentally, this can be tested by using unreactive polystyrene latex ENPs that differ in surface hydrophobicity and quartz sand grains coated with the same species of organism, but using strains which differ in their cell surface hydrophobicity.

Pseudomonas aeruginosa is a bacterium of the class gamma $(\gamma)$-proteobacteria, a wellstudied group that contains a number of human pathogens (i.e., Escherichia coli, Salmonella enterica, Yersina pestis, Vibrio cholerae, etc.). Pseudomonads are Gram negative, facultative anaerobes, non-spore-forming coccobacilli and are present ubiquitously in a diverse range of ecological niches, including granular aquatic environments, with $P$. aeruginosa PAO1 being 
used as a standard model organism in laboratory research (Stover et al., 2000). Vanhaecke et al. (1990) tested 15 different isolates of P. aeruginosa, and found P. aeruginosa PAO1 to be considerably more 'hydrophobic' than $P$. aeruginosa $9027^{\mathrm{TM}}$ in every metric used, including contact angles of $81.6^{\circ}$ versus $26.0^{\circ}$ and bacterial adherence to hydrocarbons (BATH) scores of $71-89 \%$ versus $18-19 \%$, respectively. The contrasting hydrophobicity of these isolates was confirmed in other studies (Makin \& Beveridge, 1996; Mitik-Dineva et al., 2008). Although both P. aeruginosa PAO1 and 9027TM are specifically clinical isolates (burn wound and inner-ear infections, respectively), these two two closely related bacteria are interesting candidate biofilm forming organisms to study the influence of biofilm hydrophobicity on ENP retention in saturated granular environments. present study uses clean quartz sand, coated with either $P$. aeruginosa $9027^{\mathrm{TM}}$ or PAO1, to assess the transport of two types of polystyrene latex ENPs which are also classified as either relatively hydrophilic (carboxylate-modified FluoSpheres®) or relatively hydrophobic (sulfate FluoSpheres ${ }^{\circledR}$ ) in either $\mathrm{NaCl}$ or $\mathrm{CaCl}_{2}$ at two ionic strengths (IS).

\subsection{Materials and Methods}

\subsubsection{Nanoparticle Suspensions}

Carboxylate-modified polystyrene latex $0.02 \mu \mathrm{m}$ FluoSpheres ${ }^{\circledR}$ (carboxylated latex particles; CLPs) and sulfate polystyrene latex $0.02 \mu \mathrm{m}$ FluoSpheres® (sulfate latex particles; SLPs; Life Technologies) were suspended in sodium chloride $(\mathrm{NaCl})$ or calcium chloride $\left(\mathrm{CaCl}_{2}\right)$ at 1 or 10 $\mathrm{mM}$ ionic strength (IS) and a $\mathrm{pH}$ of $7 \pm 0.05$. CLP and SLP suspensions were prepared from a stock, which was bath sonicated (Fisher Scientific FS140H, 42 $\pm 6 \mathrm{~Hz}$ ) for 2 min before dilution to $20 \mathrm{mg} / \mathrm{L}\left(\sim 2.62 \times 10^{12}\right.$ particles $\left./ \mathrm{mL}\right)$. The final suspension was then vortexed at maximum 
speed for $30 \mathrm{~s}$ to homogenize the diluted stock NPs in the suspending buffer. Suspensions were prepared 20 min before characterization or injection into the column.

\subsubsection{Characterization of Latex ENPs}

Dynamic light scattering (DLS; NanoZS Nanoseries, Malvern Instruments) was used to determine the hydrodynamic diameter ( $\left.d_{\mathrm{DLS}}\right)$ of the ENP suspensions. The $d_{\mathrm{DLS}}$ of CLPs and SLPs was measured in suspensions with a range of IS $(1,3,10,30$ and $100 \mathrm{mM})$ in $\mathrm{NaCl}$ and $\mathrm{CaCl}_{2}$ to observe their colloidal stability, or potential for aggregation, as reported in Tables $\mathrm{S} 1$ and S2. The $d_{\text {DLS }}$ of column effluent was determined on samples collected during the steady-state portion (plateau) of the breakthrough curve. Electrophoretic mobility (EPM) of column influent and effluent samples was examined using a NanoZS Nanoseries (Malvern Instruments) by laser Doppler velocimetry with an applied electrical field of $4.9 \pm 0.1 \mathrm{~V} / \mathrm{m}$ at $25^{\circ} \mathrm{C}$. The Henry equation using the Smoluchowski approximation was used to convert EPM to $\zeta$-potential. Hydrophobicity of the CLPs and SLPs were determined using a dye adsorption method adapted from Xiao and Wiesner (2012) with a hydrophilic dye and a hydrophobic dye. Briefly, $20 \mathrm{mg} / \mathrm{L}$ of Rose Bengal (hydrophobic; RB) or Nile Blue A (hydrophilic; NB) was added to CLP and SLP suspensions with a range of increasing surface area (from 0.1 to $0.001 \mathrm{~cm}^{2}$ ). Controls were prepared by adding the dyes to ultrapure water to account for the adsorption of dye to the vials and centrifuge tubes. All the samples were repeated in triplicate. After mixing and incubation in $1 \mathrm{mM} \mathrm{NaCl}(\mathrm{pH} 7.01)$ for $3 \mathrm{~h}$, CLPs or SLPs were separated from the supernatant by ultracentrifugation at $392,300 \times g$ and $4^{\circ} \mathrm{C}$ for $45 \mathrm{~min}$. The concentration of free $\mathrm{RB}$ in the supernatant was determined by UV-Vis spectroscopy at $542 \mathrm{~nm}$, while the concentration of NB was

determined at $660 \mathrm{~nm}$. The amount of dye adsorbed to the surface of the CLPs and SLPs was the 
difference between the amount of dye left in the supernatant of particle-containing conditions and the corresponding particle-free controls. Plotting the amount of each dye adsorbed to the particles as a function of total particle surface area yielded a linear regression line with a slope that is considered an indicator of the surface hydrophobicity (Xiao \& Wiesner, 2013).

\subsubsection{Characterization of Bacteria}

Modified microbial-adherence-to-hydrocarbon (MATH) tests were performed on bacterial suspensions to determine their preference for hydrophobic or hydrophilic association following a method described previously (Warne Zoueki et al., 2010) with slight modification. Briefly, $2 \mathrm{~mL}$ of washed and diluted $\left(\sim 1 \times 10^{8}\right)$ overnight culture was added to $1 \mathrm{~mL}$ dodecane and vortexed for $2 \mathrm{~min}$. Suspensions were allowed to rest for $15 \mathrm{~min}$ and the number of aqueous phase-associated bacteria was counted using a haemocytometer and phase-contrast microscopy (Olympus BX51, 40× LUCPlanFL N, NA/0.60). MATH values were determined by enumerating the initial bacterial count, subtracting the number enumerated in the aqueous phase and dividing by the initial count.

\subsubsection{Sand Preparation}

A single batch of high purity quartz sand (US-mesh size -20/+25 fraction, Granusil 2040, Ottawa plant, Unimin) washed in acid and oven dried, as described by Pelley \& Tufenkji (2008), was used for all experiments. Clean and biofilm-coated sand preparation followed the method of Mitzel \& Tufenkji (2014). Briefly, for each experimental condition, $28.5 \mathrm{~g}$ of acid washed sand was placed in a $250 \mathrm{~mL}$ glass Wheaton ${ }^{\mathrm{TM}}$ bottle (Fisher Scientific) and autoclaved for $30 \mathrm{~min}$ using a pre-vacuum cycle. For the clean sand conditions, the sterile sand was cooled to room 
temperature and $50 \mathrm{~mL}$ of the selected electrolyte solution was added to the bottle to completely cover the sand, which was left to soak for $24 \mathrm{~h}$. For each biofilm-coated condition, the same mass of sand was autoclaved and cooled to room temperature, then $15 \mathrm{~mL}$ of sterile Lysogeny Broth (LB; Fisher) was added and inoculated aseptically with either P. aeruginosa PAO1 (ATCC\# BAA-47) or P. aeruginosa 9027'TM (ATCC\# 9027) previously grown on an LB plate at $35 \pm 2{ }^{\circ} \mathrm{C}$. Uninoculated LB-coated sand served as control. Organisms were used within one week of streaking from the $-80^{\circ} \mathrm{C}$ stock. Inoculated sand batches were placed in a shaking incubator (100 rpm) and grown for $24 \mathrm{~h}$ at $35 \pm 2{ }^{\circ} \mathrm{C}$.

\subsubsection{Column Transport Experiments}

Transport experiments were performed in triplicate using glass columns (16 mm inner diameter, GE Life Sciences) and height-adjustable end-pieces in a downward flow orientation. Clean sand and biofilm-coated sand was wet packed to a length of $8.1 \mathrm{~cm}$ into glass columns using gentle vibration. Packed sand columns were equilibrated with particle-free background electrolyte for 10 pore volumes (PVs). Column effluent was monitored using a UV-visible spectrophotometer (1-cm flow-through cell, Agilent 8453) at 450, 500 and $600 \mathrm{~nm}$ wavelengths and the equilibration extended until the signals at those wavelengths remained stable for a minimum of 5 PVs, after which 4 PVs of SLPs or CLPs $(20 \mathrm{mg} / \mathrm{L})$ were injected at a rate of $0.75 \mathrm{~mL} / \mathrm{min}$ and effluent samples collected every minute.

Aliquots of the effluent were analyzed using a multi-well plate reader operating in fluorescence mode (Infinite ${ }^{\circledR} 200$ Pro NanoQuant, Tecan) by adding $200 \mu \mathrm{L}$ of each aliquot into a 96-well black microplate with transparent bottom (Fisher Scientific). The excitation was set at a wavelength of $480 \mathrm{~nm}$ and the emission at $540 \mathrm{~nm}$. These measured wavelengths differed from 
the peak intensities of the latex ENPs (ex505/em515) due to the limited band pass of the plate reader, which could not differentiate such a small difference $(10 \mathrm{~nm})$ in the peaks. Therefore, preliminary analysis was done using serial dilutions of the ENPs at various combinations of wavelengths until a reliable combination was found (i.e., ex480/em540). To ensure the reliability of experimental measurements at these modified wavelengths, a standard concentration curve was included on every analyzed microplate.

Tracer experiments were conducted separately in fresh sand columns, using either $10 \mathrm{mM}$ potassium nitrate $\left(\mathrm{KNO}_{3}\right)$ or calcium nitrate $\left(\mathrm{Ca}\left(\mathrm{NO}_{3}\right)_{2}\right)$ as tracers and monitored at $230 \mathrm{~nm}$ with a UV-visible spectrophotometer (1-cm flow-through cell, Agilent 8453). Each tracer experiment was repeated at least three times and used to obtain the effective pore volume (ePV). The ePV is defined as the time to reach the midpoint of the tracer breakthrough curve, divided by the flow rate. The nanoparticle attachment efficiency $(\alpha)$ was calculated using classical colloid filtration theory (eq 1):

$$
\alpha=-\frac{4 r_{\mathrm{C}}}{3(1-\varepsilon) \eta_{0} L} \ln \left(\frac{C}{C_{0}}\right)
$$

where $r_{\mathrm{C}}$ is the geometric radius of the collector grain, $\varepsilon$ and $L$ are the porosity and packed length of the porous medium, $C / C_{0}$ is the ratio of SLP or CLP concentration in the effluent to the influent concentration and $\eta_{0}$ is the single-collector contact efficiency, calculated using the Tufenkji-Elimelech equation (Tufenkji \& Elimelech, 2004). $C / C_{0}$ was determined by numerical integration of the breakthrough curves. The parameter values for eq 1 are listed in Table S3. Tests of significance were done using the Student's t-test $(\mathrm{P}<0.05)$ and error bars in the figures represent one standard deviation of the mean.

\subsubsection{Biofilm Coating Characterization}


A Zeiss LSM 510 META confocal laser scanning microscope (CLSM) equipped with an argon laser (458 $\mathrm{nm}$ and $488 \mathrm{~nm}$ ) was used to image the sand grain surface following transport experiments in selected conditions. Laser channel settings and stains were identical to those described previously (Mitzel \& Tufenkji, 2014). Biofilm coating on the sand grain surface was examined before injecting ENPs (pre-column biofilm) by taking sand from the top surface and the bottom of the $24 \mathrm{~h}$ incubated sand-filled bottle. Following the transport experiments, sand was taken from the 0 to $4 \mathrm{~cm}$ (termed 'Bottom') and 4 to $8 \mathrm{~cm}$ (termed 'Top') increments of the biofilm-coated sand column for imaging. Clean and LB-coated sand were investigated as negative controls, and for both, CLSM images produced no fluorescent signals, which confirms that no biofilm grew in those columns. The biofilm thickness was determined using Zen 2008 SP1.1 software as described by Mitzel \& Tufenkji (2014) and shown in the Supporting Information.

Total biomass was determined on the biofilm-coated sand from transport columns using the methods of Tripathi et al. (2012) and Mitzel \& Tufenkji (2014). Electrokinetic streaming potential analysis (Electrokinetic Analyzer, Anton Paar) was used to determine the $\zeta$-potential of the collector grains to characterize the electrokinetic properties of the clean sand, LB-coated sand and biofilm-coated sand surfaces as per Tripathi et al. (2012).

\subsection{Results and Discussion}

\subsubsection{ENP Characterization}

The transport potential of a given ENP is related to its properties, with size and charge considered to be of primary importance (Petosa et al., 2010). In the monovalent solution ( $\mathrm{NaCl}$ with IS from 1 to $100 \mathrm{mM}$ ), there was little change in the $d_{\text {DLS }}$ for either CLPs or SLPs (Tables 
$\mathrm{S} 1$ and S2). The CLP $d_{\mathrm{DLS}}$ varied from $26 \pm 2 \mathrm{~nm}$ to $28 \pm 2 \mathrm{~nm}$ and the SLP $d_{\mathrm{DLS}}$ ranged from 32 $\pm 1 \mathrm{~nm}$ to $36 \pm 1 \mathrm{~nm}$ over the range of IS, indicating the colloidal stability of both particles in $\mathrm{NaCl}$ (Fig. S1A). In the divalent salt solution $\left(\mathrm{CaCl}_{2}\right.$ with IS from 1 to $\left.100 \mathrm{mM}\right)$, the colloidal size increased more than 25 -fold with increasing IS, beginning at $10 \mathrm{mM}$ IS for CLPs and 30

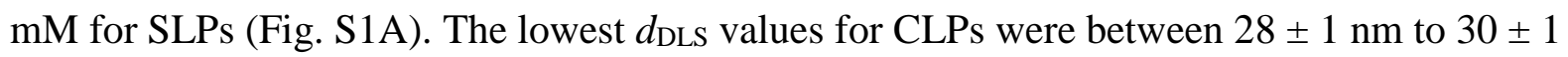
$\mathrm{nm}$ at 1 to $3 \mathrm{mM}$ IS and SLPs were between $31 \pm 1 \mathrm{~nm}$ and $36 \pm 3 \mathrm{~nm}$ in 1 to $10 \mathrm{mM} \mathrm{IS} \mathrm{CaCl}_{2}$. However, above these IS, the $d_{\text {DLS }}$ increases substantially to $805 \pm 103 \mathrm{~nm}$ for the CLPs (at 10 $\mathrm{mM}$ IS) and $1053 \pm 51 \mathrm{~nm}$ for the SPLs (at $30 \mathrm{mM}$ IS). The polydispersity index (PDI) is a dimensionless parameter calculated from cumulants analysis of the DLS-measured intensity autocorrelation function that describes the width of the assumed Gaussian distribution used in this calculation and is an indicator of the variance in the sizes of particles determined from light scattering. The PDI values for CLPs and SLPs were generally between 0.1-0.5 (suggestive of a broad monomodal distribution) over the range of conditions tested, with the exception of the SLPs at high $\left[\mathrm{CaCl}_{2}\right]$ (Fig. S1B).

The CLPs and SLPs used in this study have a reported size of $22 \pm 3 \mathrm{~nm}$ according to the manufacturer. The slightly larger average size of the SLPs in low IS solution, in comparison to the manufacturer's size, may be attributed to slight aggregation of these particles due to their surface hydrophobicity, which may render aggregation more energetically favorable in water (Morishima et al., 1989; Bowman et al., 2005). Greater particle size due to surface hydrophobicity of the SLPs is supported by the dye adsorption results presented in Table S4, which indicate that the CLPs are less hydrophobic than the SLPs. Moreover, the presence of a specific interaction between the nanoparticle surface groups (carboxylated versus sulfated) and the calcium ions may be responsible for the increased aggregation of CLPs compared to SLPs 
when suspended in $\mathrm{CaCl}_{2}$. The solubility constant of calcium carbonate $\left(K_{\mathrm{sp}}=4.5 \times 10^{-9}\right)$ in water at room temperature $\left(25^{\circ} \mathrm{C}\right)$ is much smaller than that of calcium sulfate $\left(K_{\mathrm{sp}}=2.4 \times 10^{-5}\right)$, and the heightened affinity of calcium ions for the carboxylate groups is an additional reason for the disparate particle stability behaviors observed in this study (see Fig. S1A).

The CLPs and SLPs had similar, negative $\zeta$-potentials in each salt solution, with lower $\zeta-$ potentials in the divalent than monovalent salt solutions (Fig. S1C, Tables S1 and S2). At the IS used for transport studies, CLPs were approximately $-47 \pm 3 \mathrm{mV}$ at $1 \mathrm{mM}$ and $-44 \pm 1 \mathrm{mV}$ at 10 $\mathrm{mM} \mathrm{NaCl}$ or $-20 \pm 1$ and $-18 \pm 1 \mathrm{mV}$ in 1 and $10 \mathrm{mM} \mathrm{CaCl}_{2}$, respectively. SLPs had $\zeta$-potentials of $-50 \pm 1 \mathrm{mV}$ at $1 \mathrm{mM}$ and $-56 \pm 1 \mathrm{mV}$ at $10 \mathrm{mM} \mathrm{NaCl}$ and $-21 \pm 1 \mathrm{mV}$ at $1 \mathrm{mM}$ and $-23 \pm 4$ $\mathrm{mV}$ at $10 \mathrm{mM} \mathrm{CaCl}$. Nanoparticle sizes and zeta potentials reported in Fig. S1 were determined prior to injecting suspensions into the packed columns.

\subsubsection{Characterization of the Collectors}

The transport and deposition behavior of ENPs in granular porous media depends on physicochemical particle-surface interactions (Elimelech et al., 1995; Petosa et al., 2010). Our investigation of physicochemical properties of biofilms growing on sand relied on CLSM and compound-specific dyes (e.g., FilmTracer ${ }^{\mathrm{TM}} \mathrm{FM}{ }^{\circledR}$ 1-43 Green Biofilm Cell Stain, has high binding affinity to lipids and FilmTracer ${ }^{\mathrm{TM}}$ SYPRO® Ruby Biofilm Matrix Stain, which labels primarily polysaccharides). As we were not able to directly determine the locations or molecular components on which the retained particles became attached, we have restricted our analysis of EPS components to a qualitative determination based on the appearance of the biofilms following staining. 
As seen in Fig. S2, the $P$. aeruginosa PAO1 biofilm appears to be 'greenish yellow', a qualitative indicator of higher lipid content than the P. aeruginosa $9027^{\mathrm{TM}}$ biofilm that is more 'reddish yellow', which means it has a relatively greater polysaccharide than lipid content. This interpretation is consistent with results of the modified MATH measurements (Table S6), showing that $P$. aeruginosa PAO1 displayed significantly greater hydrophobicity $\left(t_{4}=22.0, p\right.$ $<0.05$ ), with $86 \pm 3 \%$ of the cells partitioning into the hydrocarbon phase, compared to $17 \pm 5 \%$ for the $P$. aeruginosa $9027^{\mathrm{TM}}$ coating. These measurements for the planktonic bacteria indicate a clear difference in cell surface hydrophobicity between the two organisms (see Table S6) that is similar to values previously reported using the traditional MATH test (Makin \& Beveridge, 1996; Mitik-Dineva et al., 2008). Following the transport experiment, both biofilms show a relatively homogeneous coating of the sand grains without biofilm webbing extending from the grain surfaces (Fig. S2). Overall, P. aeruginosa $9027^{\mathrm{TM}}$ biofilm thickness was numerically larger, although not significantly $\left(t_{4}=1.11, p=0.32\right)$, thicker than the PAO1 biofilm (Fig. 1A). The thickness of the P. aeruginosa $9027^{\mathrm{TM}}$ biofilm was consistent regardless of column location, with a $6.7 \pm 1.6 \mu \mathrm{m}$ thick biofilm at the top and $6.7 \pm 2.0 \mu \mathrm{m}$ at the bottom. P. aeruginosa PAO1 also displayed consistent biofilm thickness throughout the column, with a biofilm thickness of $4.5 \pm 1.8 \mu \mathrm{m}$ at the top compared to $5.0 \pm 2.8 \mu \mathrm{m}$ at the bottom. We conclude that biofilm coatings on sand granules were stable over the course of the experiment and were of a similar thickness ( 4 to 5 um) to that reported by Mitzel \& Tufenkji (2014).

Biomass measurements of $P$. aeruginosa $9027^{\mathrm{TM}}$ retained within the column immediately prior to ENP exposure (Fig. 1B) show very consistent biomass, regardless of column location, with $0.6 \pm 0.1 \mathrm{mg} / \mathrm{g}$ mass at the column inlet and $0.6 \pm 0.3 \mathrm{mg} / \mathrm{g}$ at the outlet. $P$. aeruginosa PAO1 biomass was only slightly higher than the $9027^{\mathrm{TM}}$ at the top of the column, with a biomass 
of $0.8 \pm 0.4 \mathrm{mg} / \mathrm{g}$ at the column inlet. However, P. aeruginosa PAO1 biomass was significantly larger than the $9027^{\mathrm{TM}}$ at the bottom of the column, with $1.5 \pm 0.7 \mathrm{mg} / \mathrm{g}$ measured at the outlet $\left(t_{4}\right.$ $=2.37, p<0.05$ ). These results suggest that the biofilm formed by $P$. aeruginosa $9027^{\mathrm{TM}}$ was nonsignificantly thicker, but contained generally less biomass (significantly so near the outlet), therefore, the $9027^{\mathrm{TM}}$ biofilm may have been less dense than the P. aeruginosa PAO1 biofilm. This may be due to the more readily hydrated nature of the $P$. aeruginosa $9027^{\mathrm{TM}}$ biofilm, which allows more water into the biofilm as compared to the more hydrophobic, denser PAO1 biofilm. The greater thickness from increased hydration would not have been accounted for in the biomass determination that was done by drying and combustion. It should be noted that accounting for the non-significant differences in biofilm thickness would only change the calculated attachment efficienciesby less than $1 \%$ in every condition (calculations not shown).The biomass retained in the column following the transport experiment was approximately 2 to 20 times greater in this study than in other experiments with biofilm-coated sand conducted with various organisms and growth conditions (Lerner et al., 2012; Tripathi et al., 2012; Jiang et al., 2013; Xiao \& Wiesner, 2013).

Despite the difference in biofilm biomass, our measurements of the effective pore volume (ePV) of the columns revealed that tracer curves had the same shape and breakthrough occurred at similar times ( 400 sec), indicating a similar ePV in clean sand and biofilm-coated sand columns (Fig. S3). Specifically, both the P. aeruginosa PAO1 and 9027 had ePVs of $6.0 \pm 0.1 \mathrm{~mL}$ in monovalent salt and $6.1 \pm 0.1 \mathrm{~mL}$ in divalent salt and similar porosities (Table S3). Also, the charge of the biofilm coatings was similar between the two investigated bacterial strains at $-20.4 \pm 0.2 \mathrm{mV}$ and $-12.5 \pm 0.8 \mathrm{mV}$ for the P. aeruginosa PAO1 coating, and $-20.2 \pm 0.4 \mathrm{mV}$ and $-12.1 \pm 0.5 \mathrm{mV}$ for $P$. aeruginosa $9027^{\mathrm{TM}}$ at $1 \mathrm{mM} \mathrm{NaCl}$ and 
$\mathrm{CaCl}_{2}$, respectively (Table S5). The $\zeta$-potentials become less negative as IS increased to $10 \mathrm{mM}$ $\mathrm{NaCl}$ and $\mathrm{CaCl}_{2}$, rising to $-15.5 \pm 0.6 \mathrm{mV}$ and $-9.3 \pm 0.3 \mathrm{mV}$ for the $P$. aeruginosa $\mathrm{PAO} 1$ coating and $-13.5 \pm 0.6 \mathrm{mV}$ and $-8.0 \pm 0.2 \mathrm{mV}$ for $P$. aeruginosa $9027^{\mathrm{TM}}$, respectively. In salt solutions with IS of $10 \mathrm{mM}$, the $9027^{\mathrm{TM}}$ biofilm-coated sand was significantly less negatively charged than the PAO1-coated sand, in both $\mathrm{NaCl}\left(t_{4}=4.08, p<0.05\right)$ and $\mathrm{CaCl}_{2}\left(t_{4}=6.25, p<0.05\right)$. In addition, the $P$. aeruginosa $9027^{\mathrm{TM}}$ and PAO1 biofilm-coated sands had significantly $(\mathrm{p}<0.05)$ more negative charge (about -2 to $-4 \mathrm{mV}$ lower) than the clean sand in both monovalent and divalent salt solutions at all IS. Due to the inherent theoretical limitations in the measurement of $\zeta$-potential of a biofilm (a soft, inhomogeneous film), statistical significance should be interpreted with care.

\subsubsection{Transport of CLPs and SLPS}

When suspended at low IS of either mono- or divalent salt, both CLPs and SLPs experienced little retention in the clean sand columns. By comparison, increased particle retention was consistently observed in the biofilm-coated sands. Most notably, there appeared to be differences between the hydrophilic and hydrophobic biofilm conditions, particularly with increasing IS. At low IS $\mathrm{NaCl}$ (Fig. 2A and 3A), the slopes of the rising limb of the breakthrough curves for the clean sand and both $P$. aeruginosa $\mathrm{PAO}$ and $9027^{\mathrm{TM}}$ biofilm-coated sands appear similar; ultimately rising to a higher plateau (i.e., less steady-state retention) in the $9027^{\mathrm{TM}}$ biofilm than the PAO1 biofilm. However, at $10 \mathrm{mM} \mathrm{NaCl}$ and $1 \mathrm{mM} \mathrm{CaCl}_{2}$ (Fig. 2 and 3, B and C), the initial slopes of the breakthrough curves differ noticeably between the two biofilm coatings. The breakthrough curves for the $P$. aeruginosa PAO1 remain similar to those measured in $1 \mathrm{mM}$ $\mathrm{NaCl}$, but the shape of the $P$. aeruginosa $9027^{\mathrm{TM}}$ breakthrough curves become considerably less 
steep; rising slowly over the course of the nanoparticle injection. Inspection of the breakthrough curves reveals that this behavior is independent of the type of nanoparticle. These differences suggest that the dynamics of nanoparticle deposition onto a hydrophilic biofilm can be fundamentally different than the deposition of nanoparticles onto a hydrophobic biofilm, as the observed nanoparticle deposition kinetics are variably responsive to changes in IS between these two biofilm types. At $10 \mathrm{mM} \mathrm{CaCl}_{2}$, the SLPs displayed considerable elution in clean sand and nearly complete retention in both types of biofilm-coated sand. At this IS of divalent salt, CLPs are above their critical coagulation concentration (CCC) and the extensive particle aggregation leads to significant retention in all three sand treatments.

To allow for semi-quantitative comparison of nanoparticle transport properties, the nanoparticle attachment efficiencies $(\alpha)$ were determined at 1 and $10 \mathrm{mM}$ IS of the two electrolytes for the clean, P. aeruginosa $9027^{\mathrm{TM}}$ and $P$. aeruginosa $\mathrm{PAO1-coated} \mathrm{sand} \mathrm{grains}$ (Fig. S4). At low IS of both $\mathrm{NaCl}$ and $\mathrm{CaCl}_{2}$, the clean sand surface yielded the lowest $\alpha$ for both SLPs and CLPs, ranging from $10^{-4}$ to $10^{-3}$. In comparison, both P. aeruginosa coatings yielded larger values of $\alpha$ for the two nanoparticles, on the order of $10^{-2}$ to $10^{-1}$, indicating that both types of biofilm increased retention of the electrostatically-stabilized latex nanoparticles compared to clean sand. Despite the visibly different breakthrough curves mentioned previously, there were only minor differences in the overall calculated attachment efficiency between the two types of biofilm coating, with both biofilm types displaying similar $\alpha$ values at low IS in both salts. Retention profiles and recovery of the latex ENPs were not determined due to the possible confounding influence of the biomass.

The size $\left(d_{\mathrm{DLS}}\right)$ and charge $(\zeta$-potential) of nanoparticles in the column effluent are presented in Tables $\mathrm{S} 2$ and $\mathrm{S} 3$. In 1 and $10 \mathrm{mM} \mathrm{NaCl}$, as well as $1 \mathrm{mM} \mathrm{CaCl}_{2}$, there is little to no 
difference in the influent versus effluent $d_{\mathrm{DLS}}(\sim 30 \mathrm{~nm})$ indicating that the CLPs and SLPs remained stable in suspension and that there was no size-dependent filtration of either nanoparticle at these solution chemistries. In $10 \mathrm{mM}$ IS $\mathrm{CaCl}_{2}$, the SLPs remained generally stable against aggregation; however, the CLPs in $10 \mathrm{mM}$ IS $\mathrm{CaCl}_{2}$ displayed extensive destabilization leading to very large $d_{\mathrm{DLS}}(\sim 800 \mathrm{~nm})$. Accordingly, the CLPs in $10 \mathrm{mM} \mathrm{IS} \mathrm{CaCl} 2$ exhibited $\alpha$ values considerably greater than $1(\sim 5)$ in $10 \mathrm{mM} \mathrm{CaCl}_{2}$, in all three of the sand treatments, indicating that a physical straining mechanism was contributing to the particle retention behavior at this condition (Bradford et al., 2002; Raychoudhury et al., 2014).

Research using charged (hydrophilic, poly-acrylic acid) and uncharged (more hydrophobic, poly-ethylene oxide) polymer brushes has shown that hydrophobic polymer coatings readily shift to a 'block' formation (i.e., a dense, film-like state) with increased mechanical confinement, while hydrophilic polymer coatings display a more gradual shift to a block formation (Abbott et al. 2015). Hydrophobic polymer brushes were, conversely, much more resistant ( 100 times) to osmotic pressure confinement than mechanical confinement (Hansen et al., 2003), while hydrophilic brushes displayed a nonmonotonic behaviour (i.e., they first swell, then shrink) as a function of increasing IS (Currie et al., 2000). A biofilm is much more complex than a polymer brush, containing a network of pores likely lined with a complex, heterogeneous network of polymers and other biomaterials. It is unclear how relevant a comparison polymer brushes are to biofilms that would differ considerably in the distribution of surface moieties. Biofilms also contain an internal void space with properties distinct from that of the surface (Costerton et al., 1995). Nonetheless, a similar observation was made in this study, in that greater IS-induced retention was observed in the hydrophilic biofilm condition, indicating a preferential effect for ionic destabilization of hydrophilic, compared to hydrophobic biofilms. 
These previous findings may be taken as evidence to support the idea that hydrophobic biofilms may have some quality of their polymeric component (e.g., extracellular polymeric substances; EPS) that stabilizes them against the effects of increasing IS.

The significantly different $\zeta$-potentials of the biofilms at higher IS may have also impacted the transport of the charged SLPs and CLPs. At $10 \mathrm{mM} \mathrm{NaCl}$, where a significant difference in charge was observed, less retention of both types of polystyrene nanoparticles occurred with the more negative $P$. aeruginosa PAO1 biofilm. In $10 \mathrm{mM} \mathrm{CaCl}_{2}$, the retention of both particles in both biofilms is very high and the observed differences in biofilm charge are not directly reflected in the breakthrough curves as the electrostatic repulsion is minimal at this high IS of divalent salt.

Based on the findings of this study, particle surface hydrophobicity does not seem to be a major determinant of the overall transport potential of nanoparticles since the measured breakthrough curves for the two types of nanoparticles were generally comparable. It appears, from this work and previous research, that the type of stabilization method of the ENPs (i.e., steric vs. electrostatic) and whether the surface is clean or coated with biofilm are factors of greater importance. The current study did not investigate whether the nanoparticles attached to the surface of the biofilm or if they were retained within the biofilm pores; and these may be factors in the observed variation in retention. Habimana et al. (2011) reported that similar CLPs to those used here, but of a slightly larger size $(50 \mathrm{~nm})$, could enter a biofilm of Lactococcus lactis. The distinction between nanoparticles entering a biofilm or sticking on the surface may have pertinent implications regarding the transport behavior of nanoparticles, as well as major toxicological implications. Future research in our laboratory will focus on elucidating these differences and the exact nature of nanoparticle deposition onto or into the biofilm matrix. It 
should also be noted that the ENPs used in this study are largely considered chemically inert, in that they do not release a dissolved component (unlike $\mathrm{Ag}^{+}$released by $n \mathrm{Ag}$ or Fe ions released by zerovalent iron nanoparticles) into the suspending media (Basnet et al., 2016). As many other ENPs do release dissolved components, the transport of these types of ENPs may be complicated by the interactions between biofilms and ENP-associated dissolved components.

We have also presented evidence in this study that changing the hydrophobicity of the biofilm coating results in significant modification of the dynamics of retention. The difference in deposition dynamics between hydrophobic and hydrophilic biofilms may be important factors for transport and persistence of nanoparticles following environmental release. Thus, future work can focus on the transport behavior of nanoparticles in more complex, mixed species biofilmcoated sands.

\subsection{Conclusions}

- Measurable differences in particle surface hydrophobicity of sulfate (SLPs) and carboxylate (CLPs) functionalized latex nanoparticles were detected using a dyeadsorption assay. SLPs were more resistant to divalent ionic strength destabilization, either due to hydrophobicity of the SLPs or lower affinity for $\mathrm{Ca}^{2+}$ ions.

- Biofilm-coated sand had significantly greater retention of electrostatically-stabilized latex nanoparticles than clean sand.

- Breakthrough curves illustrated that nanoparticle breakthrough in the P. aeruginosa PAO1 biofilm-coated sand column was generally constant with time and breakthrough in the $P$. aeruginosa 9027 biofilm-coated sand column showed dynamic behavior. 
- Increasing IS did not modify nanoparticle retention by the hydrophobic biofilm, but resulted in variable retention of the nanoparticles by the hydrophilic biofilm. 


\section{Acknowledgements}

The authors acknowledge the financial support of NSERC, Environment Canada, the Ministère du Développement économique, Innovation et Exportation (MDEIE), the Concordia Institute for Water, Energy and Sustainable Systems for a CREATE award, and McGill University for the F. Grey Woods Fellowship to MRM. 


\section{References}

Abbott, S. B., de Vos, W. M., Mears, L. L., Cattoz, B., Skoda, M. W., Barker, R., Richardson, R. M., Prescott, S. W., 2015. Is osmotic pressure relevant in the mechanical confinement of a polymer brush?. Macromolecules 48 (7), 2224-2234.

Basnet, M., Gershanov, A., Wilkinson, K. J., Ghoshal, S., Tufenkji, N., 2016. Interaction between palladiumdoped zerovalent iron nanoparticles and biofilm in granular porous media: characterization, transport and viability. Environmental Science: Nano. DOI: 10.1039/C5EN00109A

Bradford, S. A., Yates, S. R., Bettahar, M., Simunek, J., 2002. Physical factors affecting the transport and fate of colloids in saturated porous media. Water Resources Research 38 (12), 63-1.

Brar, S. K., Verma, M., Tyagi, R. D., Surampalli, R. Y. 2010. Engineered nanoparticles in wastewater and wastewater sludge-Evidence and impacts. Waste Management 30 (3), 504-520.

Chandler, D., 2005. Interfaces and the driving force of hydrophobic assembly. Nature 437 (7059), 640-647.

Cornelis, G., Hund-Rinke, K., Kuhlbusch, T., Van den Brink, N., Nickel, C., 2014. Fate and bioavailability of engineered nanoparticles in soils: a review. Critical Reviews in Environmental Science and Technology 44 (24), 2720-2764.

Costerton, J. W., Lewandowski, Z., Caldwell, D. E., Korber, D. R., Lappin-Scott, H. M., 1995. Microbial biofilms. Annual Reviews in Microbiology 49 (1), 711-745.

Currie, E. P. K., Sieval, A. B., Fleer, G. J., Stuart, M. C., 2000. Polyacrylic acid brushes: surface pressure and salt-induced swelling. Langmuir 16 (22), 8324-8333.

Donlan, R. M. 2002. Biofilms: microbial life on surfaces. Emerging Infectious Diseases, 8 (9), DOI: 10.3201/eid0809.020063.

Elimelech, M., Gregory, J., Jia, X., Williams, R A., 1995. Particle Deposition and Aggregation, Measurement, Modeling and Simulation, $10^{\text {th }}$ Edition. Butterworth-Heinemann, Waltham, MA.

Flemming, H. C., Wingender, J., 2010. The biofilm matrix. Nature Reviews Microbiology 8 (9), 623-633.

Habimana, O., Steenkeste, K., Fontaine-Aupart, M. P., Bellon-Fontaine, M. N., Kulakauskas, S., Briandet, R., 2011. Diffusion of nanoparticles in biofilms is altered by bacterial cell wall hydrophobicity. Applied and Environmental Microbiology 77 (1), 367-368.

Hansen, P. L., Cohen, J. A., Podgornik, R., Parsegian, V. A., 2003. Osmotic properties of poly (ethylene glycols): quantitative features of brush and bulk scaling laws. Biophysical Journal 84 (1), 350-355.

Israelachvili, J., Pashley, R., 1982. The hydrophobic interaction is long range, decaying exponentially with distance. Nature 300 (5890), 341-342.

Israelachvili, J., Wennerström, H., 1996. Role of hydration and water structure in biological and colloidal interactions. Nature 379 (6562), 219-225.

Jiang, X., Wang, X., Tong, M., Kim H., 2013. Initial transport and retention behaviors of ZnO nanoparticles in quartz sand porous media coated with Escherichia coli biofilm. Environmental Pollution 174, 38-49.

Kanduč, M., Schneck, E., Netz, R. R., 2014. Attraction between hydrated hydrophilic surfaces. Chemical Physics Letters 610, 375-380.

Keller, A. A., McFerran, S., Lazareva, A., Suh, S., 2013. Global life cycle release of engineered nanomaterials. Journal of Nanoparticle Research 15 (1692), 1-17.

Leon-Morales, C. F., Leis, A. P., Strathmann, M., Flemming, H-C., 2007. Interactions between laponite and microbial biofilms in porous media: implications for colloid transport and biofilm stability. Water Research 38, 3614-3626.

Lerner, R. N., Lu, Q., Zeng, H., Liu, Y., 2012. The effects of biofilm on the transport of stabilizes zerovalent iron nanoparticles in saturated porous media. Water Research 46 (4), 975-985. 
Lin, D., Tian, X., Wu, F., Xing, B., 2010. Fate and transport of engineered nanomaterials in the environment. Journal of Environmental Quality 39, 1896-1908.

Lin, S., Cheng, Y., Liu, J., Wiesner, M. R. 2012. Polymeric coatings on silver nanoparticles hinder autoaggregation but enhance attachment to uncoated surfaces. Langmuir 28 (9), 4178-4186.

Lowry, G. V., Gregory, K. B., Apte, S. C., Lead, J. R., 2012. Transformations of nanomaterials in the environment. Environmental Science and Technology 46 (13), 6893-6899.

Makin, S. A., Beveridge, T. J., 1996. The influence of A-band and B-band lipopolysaccharide on the surface characteristics and adhesion of Pseudomonas aeruginosa to surfaces. Microbiology 142, 299-307.

Mitik-Dineva, N., Wang, J., Khanh-Truong, V., Stoddart, P., Malherbe, F., Crawford, R. J., Ivanova, E. P., 2009. Escherichia coli, Pseudomonas aeruginosa, and Staphylococcus aureus attachment patterns on glass surfaces with nanoscale roughness. Current Microbiology 142, 299-307.

Mitzel, M. R., Tufenkji, N., 2014. Transport of industrial PVP-stabilized silver nanoparticles in saturated quartz sand coated with Pseudomonas aeruginosa PAO1 biofilm of variable age. Environmental Science and Technology 48 (5), 2715-2723.

Morishima, Y., Kobayashi, T., Nozakura, S., 1989. Amphiphilic polyelectroytes with various hydrophobic groups: intramolecular hydrophobic aggregation in aqueous solution. Polymer Journal 21 (3), 267274.

Musee N., 2011. Simulated environmental risk estimation of engineered nanomaterials: a case of cosmetics in Johannesburg City. Human and Experimental Toxicology 30, 1181-1195.

Petosa, A. R., Jaisi, D. P., Quevedo, I. R., Elimelich, M., Tufenkji, N., 2010. Aggregation and deposition of engineered nanomaterials in aquatic environments: role of physicochemical interactions. Environmental Science and Technology 44 (17), 6532-6549.

Petosa, A. R.; Brennan, S. J.; Rajput, F.; Tufenkji, N., 2012. Transport of two metal oxide nanoparticles in saturated granular porous media: role of water chemistry and particle coating. Water Research 46 (4), 1273-1285.

Raychoudhury, T., Tufenkji, N., Ghoshal, S., 2014. Straining of polyelectrolyte-stabilized nanoscale zerovalent iron particles during transport through granular porous media. Water Research 50, 80-89.

Sabitha, M., Jose, S., Raj, S., Sumod, U., 2012. Nanotechnology in cosmetics: opportunities and challenges. Journal of Pharmacy and Bioallied Sciences 4, 186-193.

Saleh, N., Kim, H. J., Phenrat. T., Matyjaszewski, K., Tilton, R. D., Lowry, G. V., 2008. Ionic trength and composition affect the mobility of surface-modified $\mathrm{Fe} 0$ nanoparticles in water-saturated sand columns. Environmental Science and Technology 42, 3349-3355.

Song, J. E., Phenrat, T., Marinakos, S., Xiao, Y., Liu, J., Wiesner, M. R., Tilton, R. D., Lowry, G. V., 2011. Hydrophobic interactions increase attachment of gum arabic-and PVP-coated Ag nanoparticles to hydrophobic surfaces. Environmental Science and Technology 45, 5988-5995.

Stover, C. K., Pham, X. Q., Erwin, A. L., Mizoguchi, S. D., Warrener, P., Hickey, M. J., Brinkman, F. S. L., Hufnagle, W. O., Kowalik, D. J., Lagrou, M., Garber, R. L.. Goltry, L., Tolentino, E., WestbrockWadman, S., Yuan, Y., Brody, L. L., Coulter, S. N., Folger, K. R., Kas, A., Larbig, K., Lim, R., Smith, K., Spencer, D., Wong, G. K-S., Wu, Z., Paulsen, I. T., Reizer, J., Saier, M. H., Hancock, R. E. W., Lory, S., Olson, M. V., 2000. Complete genome sequence of Pseudomonas aeruginosa PAO1, an opportunistic pathogen. Nature 406, 959-964.

Tong, M., Ding, J., Shen, Y., Zhu, P., 2010. Influence of biofilm on the transport of fullerene (C60) nanoparticles in porous media. Water Research 44 (4), 94-103.

Tufenkji, N., Elimelech, M., 2004. Correlation equation for predicting single-collector efficiency in physicochemical filtration in saturated porous media. Environmental Science and Technology 38, 529536. 
Tripathi, S., Champagne, D., Tufenkji, N., 2012. Transport behavior of selected nanoparticles with different surface coating in granular porous media coated with Pseudomonas aeruginosa biofilm. Environmental Science and Technology 46, 6942-6949.

Vanhaecke, E., Remon, J., Moors, M., Reas, F., Rudder, D., van Peteghem, A., 1990. Kinetics of Pseudomonas aeruginosa adhesion to 304 and 316-L stainless steel: role of cell surfaces hydrophobicity. Applied and Environmental Microbiology 56 (3), 788-795.

Watnick, P., Kolter, R., 2000. Biofilm, City of Microbes. Journal of Bacteriology 182 (10), 2675-2679.

Xiao, Y., Wiesner, M. R., 2012. Characterization of surface hydrophobicity of engineered nanoparticles. Journal of Hazardous Materials, 215, 146-151.

Xiao, Y., Wiesner, M. R., 2013. Transport and retention of selected engineered nanoparticles by porous media in the presence of biofilm. Environmental Science and Technology 47, 2246-2253.

Zoueki, C. W., Tufenkji, N., Ghoshal, S., 2010. A modified microbial adhesion to hydrocarbons assay to account for the presence of hydrocarbon droplets. Journal of Colloid and Interface Science 344 (2), 492-496. 
Figures
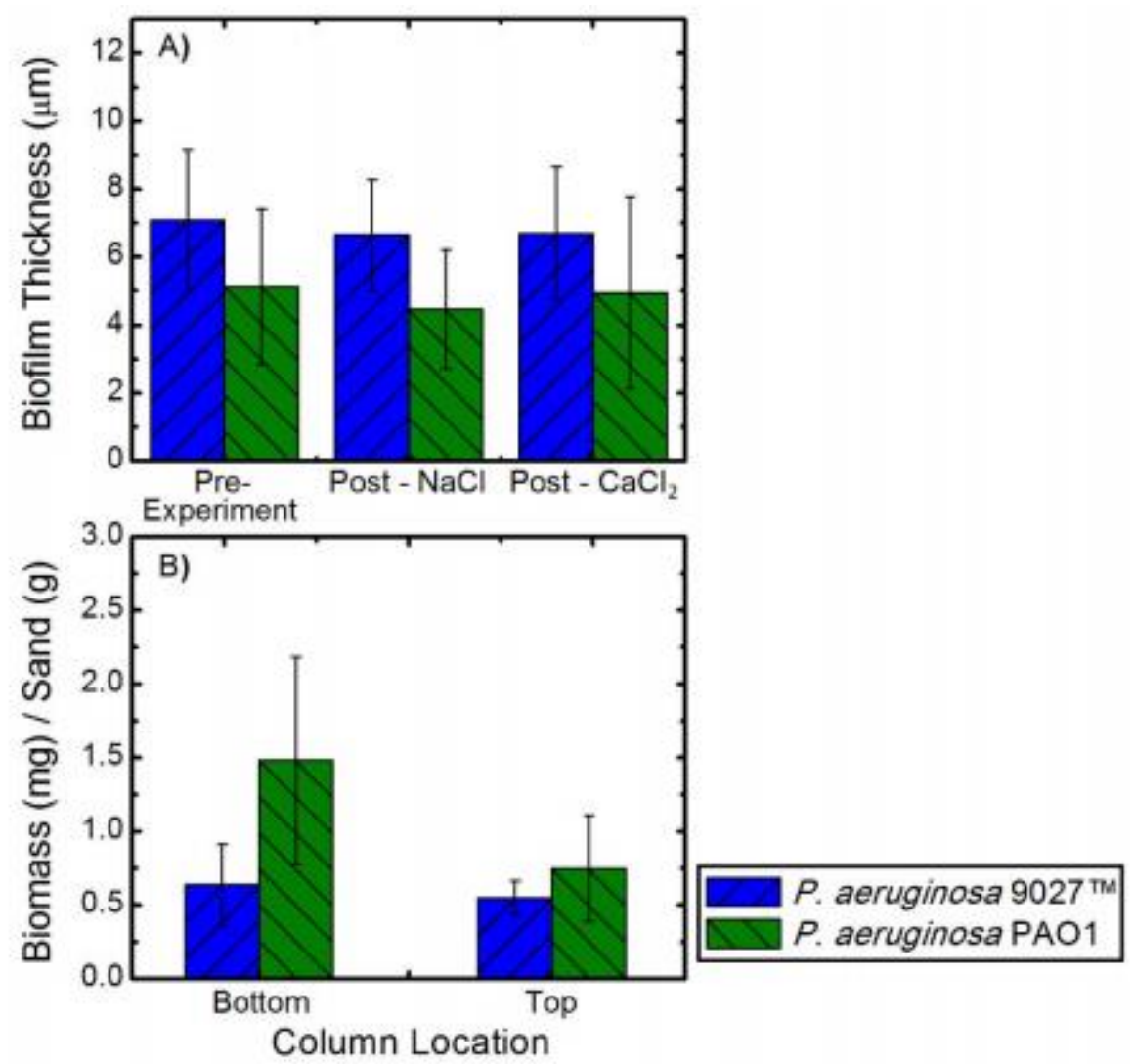

Figure 1. (A) Average biofilm thickness as measured for the biofilm taken directly from the growth bottle (pre-experiment) and after equilibration using $\mathrm{NaCl}(\mathrm{Post} \mathrm{NaCl})$ or $\mathrm{CaCl}_{2}(\mathrm{Post}$ $\mathrm{CaCl}_{2}$ ) and (B) biomass distribution for both $P$. aeruginosa PAO1 and $9027^{\mathrm{TM}}$ strains from the top and bottom of post-equilibration with $\mathrm{NaCl}$ (Post $\mathrm{NaCl}$ ) column. Error bars represent 1 standard deviation. 

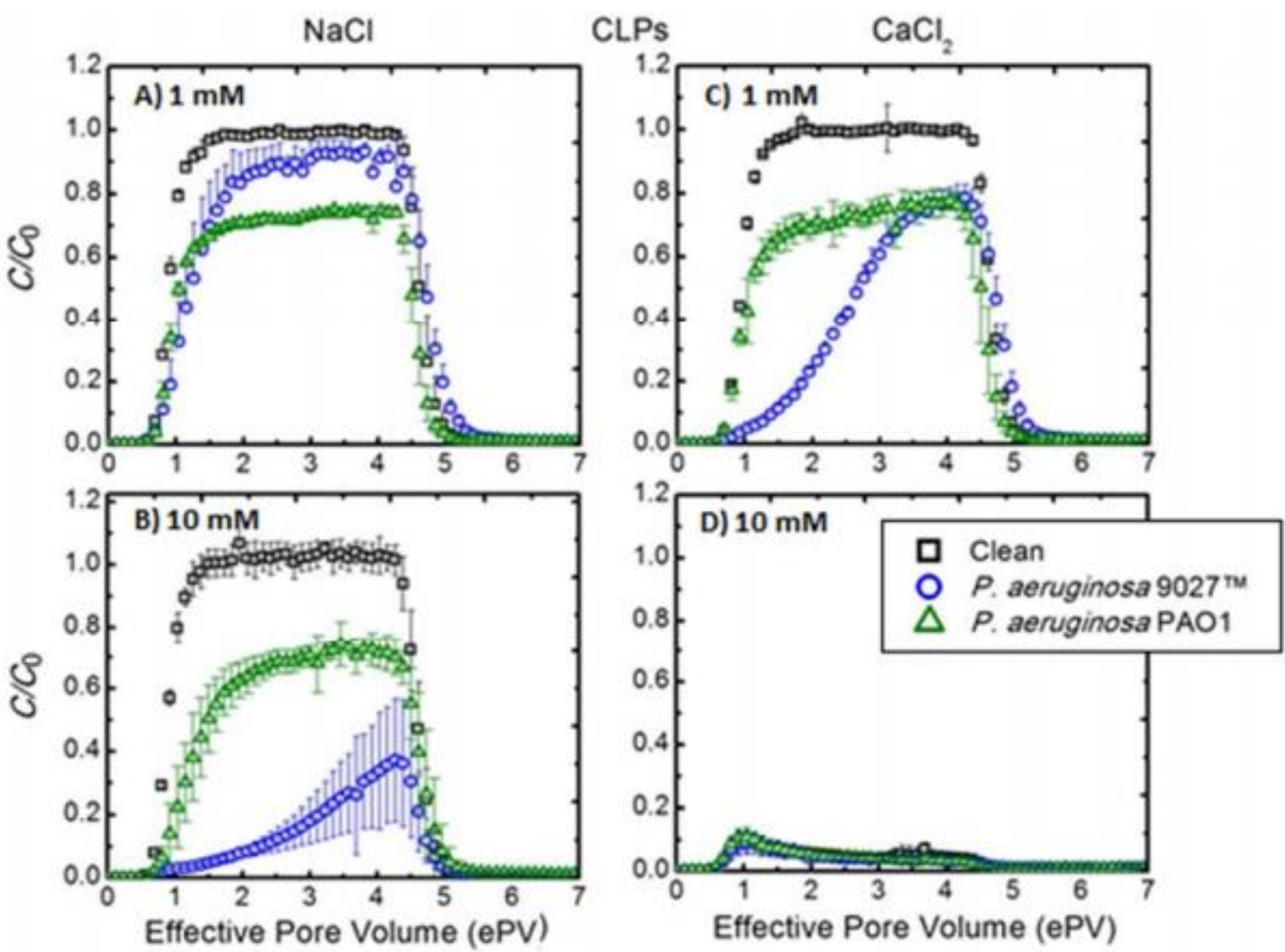

Figure 2. Breakthrough curves of carboxylate-modified latex Fluospheres $\mathbb{R}$ (CLPs) in (A) $1 \mathrm{mM}$ and (B) $10 \mathrm{mM} \mathrm{NaCl}$ and (C) $1 \mathrm{mM}$ and (D) $10 \mathrm{mM}$ ionic strength $\mathrm{CaCl}_{2}$ using clean sand (black squares), $P$. aeruginosa $9027^{\mathrm{TM}}$ (blue circles) and $P$. aeruginosa PAO1 (green triangles). Error bars represent 1 standard deviation. 

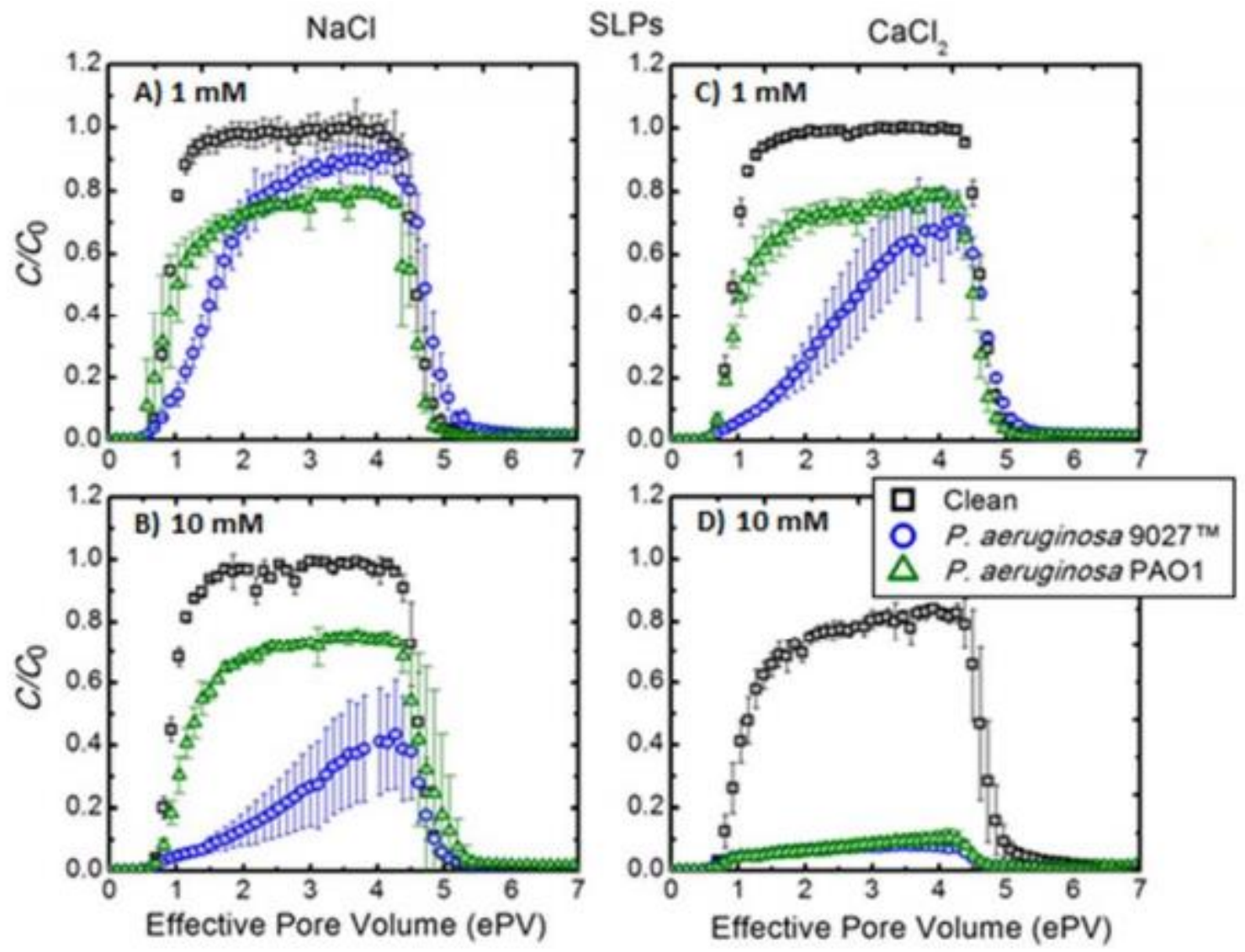

Figure 3. Breakthrough curves of sulfate latex Fluospheres $($ SLPs) in $1 \mathrm{mM}$ (A) and $10 \mathrm{mM}$ (B) $\mathrm{NaCl}$ and $1 \mathrm{mM}$ (C) and $10 \mathrm{mM}$ (D) ionic strength $\mathrm{CaCl}_{2}$ using clean sand (black squares), P. aeruginosa $9027^{\mathrm{TM}}$ (blue circles) and P. aeruginosa PAO1 (green triangles). Error bars represent 1 standard deviation. 